TITRE: INTERNET ET MÉDIAS TECHNOLOGIQUES, OUTILS COGNITIFS DE MÉDIATION DU SAVOIR EN CLASSE DE FRANÇAIS DANS UN CONTEXTE DE RECHERCHE-ACTION

Auteur(s): Athanase Simbagoye, Ph.D., Université Laurentienne

PublicATION: CRÉATION DE DISPOSITIFS DIDACTIQUES ET ENSEIGNEMENT-APPRENTISSAGE DIVERSIFIÉ EN LITTÉRATIE : VERS UNE VALORISATION DE LA RECHERCHE-DÉVELOPPEMENT ET DE LA RECHERCHEACTION EN ÉDUCATION

PAGES: $58-75$

Directeurs: Marie-Christine BeAudry, ISABelle Carignan et FrançoIS LAROSE

ÉditeUR: LES ÉDITIONS DE L'UNIVERSITÉ DE SHERBROOKE, 2017

ISBN: 978-2-7622-0356-1

URI: HTTP://HDL.HANDLE.NET/11143/10125

DOI: HTTP://DX.DOI.ORG/10.17118/11143/10125 


\title{
INTERNET ET MÉDIAS TECHNOLOGIQUES, OUTILS COGNITIFS DE MÉDIATION DU SAVOIR EN CLASSE DE FRANÇAIS DANS UN CONTEXTE DE RECHERCHE-ACTION
}

\author{
Athanase Simbagoye, Ph.D., Université Laurentienne
}

Résumé : Le texte présente les résultats d'une recherche-action menée dans le cadre d'un projet pilote portant sur l'intégration des TIC dans les apprentissages de base (français, langue seconde et mathématique) à l'école élémentaire au Sénégal. L'auteur y montre comment l'intégration des TIC aux activités de français transforme les pratiques pédagogiques des enseignants et les stratégies d'apprentissage de la langue chez les élèves. Il esquisse également les fondements d'une didactique situationnelle à la base du développement des compétences langagières chez les élèves qui apprennent une langue seconde.

Mots-clés : construction, littératie, recherche-action, situation, technologie 


\section{Introduction}

Ce texte présente les résultats d'une recherche-action menée dans le cadre d'un projet pilote portant sur l'intégration des technologies de l'information et de la communication (TIC) dans les apprentissages de base (français et mathématique) à l'école Serigne Amadou Aly Mbaye (SAAM) de Dakar, au Sénégal. C'est dans un contexte d'accompagnement des enseignants à l'intégration des TIC dans les apprentissages de base à l'élémentaire qu'une équipe de chercheurs de la Chaire UNESCO de Développement curriculaire de l'Université du Québec à Montréal (CUDC) s'est jointe, de 2003 à 2012, aux chercheurs sénégalais de l'Institut national d'étude et d'action pour le développement de l'éducation (INÉADE) pour mener un projet de recherche collaborative. Le projet comportait quatre phases: «(1) démarrage; (2) coconstruction des situations pédagogiques; (3) expérimentation des situations; et (4) diffusion » (Munger et Jonnaert, 2007, p. 12). Ce texte présente une analyse des données recueillies à la $3^{e}$ phase. À la lumière des résultats provenant des observations de séquences d'enseignement-apprentissage du français en 5e année et des entrevues réalisées avec des enseignants expérimentateurs, l'auteur analyse les fondements d'une didactique situationnelle qui faciliterait le développement des compétences en littératie scolaire chez les élèves qui apprennent le français, langue de scolarisation. Le développement du texte répond à la question suivante : quel est l'impact de la technologie sur la qualité des apprentissages en français, langue de scolarisation, à l'école primaire? Cette question s'est posée durant la phase 3, et ce, dans le prolongement d'une autre question soulevée au moment de la coconstruction des situations pédagogiques : comment exploiter la technologie pour faciliter les apprentissages en français, langue de scolarisation, à l'école primaire? Le but de l'intégration des TIC était celui de développer des compétences en littératie chez l'élève. Autrement dit, l'approche didactique privilégiée dans le projet visait la planification d'une variété de situations d'enseignement et d'apprentissage intégrant des outils médiatiques ayant un potentiel cognitif élevé pour améliorer la qualité des apprentissages. Notre argumentaire s’appuie sur des écrits qui relèvent du paradigme socioconstructiviste et de celui de la cognition située pour décrire les résultats de la recherche.

\section{Cadre conceptuel}

Depuis quelques décennies, le paradigme constructiviste sert de cadre théorique de référence dans des études en éducation. Le constructivisme concerne la question épistémologique du rapport de l'être humain au monde et au savoir ainsi que du mode de constitution des connaissances chez l'humain. Le paradigme constructiviste concède à la personne le rôle premier dans la construction de ses propres connaissances. Du point de vue de quelques-unes de ses traditions, le constructivisme soutient le point de vue selon lequel le sujet humain crée son monde tout en se créant lui-même (Jonnaert, 2004; Maturana, 1998; Von Glasersfeld, 1999, 2004). Dans cette perspective, deux indications importantes doivent être soulignées. La première est que l'être humain est responsable de sa pensée, de sa connaissance, et donc de ce qu'il fait (Von Glasersfeld, 2004). Il est le dépositaire de 
ses connaissances et celles-ci émanent par conséquent de son action. La deuxième indication est celle selon laquelle la construction de son monde par l'humain procède d'une organisation d'actions, processus au moyen duquel celui-ci et son monde émergent ensemble. Le sujet humain trouve dans cette émergence les conditions de s'actualiser et de se produire par le biais de sa disposition d'esprit, de sa réflexion dans l'action et de son savoir agir (Roth et Masciotra, 2004). Cependant, tout sujet humain a une histoire et elle influe sur lui. Il y a donc une dimension socioculturelle qu'on aurait tort de ne pas considérer dans le processus d'apprentissage. C'est la perspective sociologique du constructivisme (Vygotski, 1985) dont s'inspirent les tenants de l'approche par les situations aujourd'hui privilégiée par les réformes curriculaires axées sur le développement de compétences (Jonnaert et van der Borgh, 2003). Cette perspective prend en compte le point de vue vygotskien concernant le caractère social de l'apprentissage et de l'inévitable détermination culturelle de celui-ci. La dimension socioculturelle de l'apprentissage tient compte du caractère situé du processus d'apprentissage (Brown, Collins et Duguid, 1989; Lave, 1991; Lave et Wenger, 1991) et de l'existence d'une communauté d'apprentissage. D'un côté, le caractère situé de l'apprentissage réfère à l'idée que la cognition, l'activité du sujet et le contexte de l'apprentissage sont inséparables. D'un autre côté, l'idée de communauté d'apprentissage a des similarités avec le concept de communauté de pratique (Wenger, 1998) au plan pragmatique. Ces aspects sont importants pour comprendre l'approche par les situations d'enseignement-apprentissage qui tiennent compte de la culture et du contexte de vie de l'élève. Ils permettent de comprendre l'accent ayant été mis sur l'importance des interactions langagières en classe et lors des activités extramuros durant ce projet de recherche-action intégrant les TIC à l'école Serigne Amadou Aly Mbaye (SAAM) de Dakar.

\section{Méthodologie}

Dans le cadre du projet d'intégration des TIC dans les apprentissages de base, l'école SAAM a été dotée de 25 ordinateurs installés dans quatre classes expérimentales et d'une connexion Internet haute vitesse. Chaque classe comptait 36 élèves répartis en équipes de travail mixtes de six élèves. Pour mieux intégrer les TIC en français et en mathématiques, la pédagogie du projet est adoptée. Plusieurs projets intégrateurs (PI), projets didactiques ayant comme visée l'amélioration des pratiques pédagogiques, ont ainsi été vécus. Ainsi, il y avait déjà des acquis du projet au niveau des apprentissages au moment la cueillette de données (à la phase 3 ) dont nous présentons les résultats dans cet article. Les PI avaient donc permis la réalisation de deux projets (péril fécal et vers intestinaux) par des élèves (Jonnaert, 2010). Les focus groups et les entrevues réalisées avec les enseignants à la phase 3 montrent que leur contenu fait écho aux acquis du projet. Nous y reviendrons en illustrant cela en recourant à quelques témoignages recueillis à cette étape-là. 
Durant la période d'implantation de l'outil informatique (phase de démarrage), les enseignants des classes expérimentales reçoivent une série de formations portant sur l'utilisation de l'ordinateur et sur l'exploitation des possibilités qu'offre l'Internet dans le contexte de l'enseignement du français (par exemple, consulter le dictionnaire en ligne, faire de la recherche documentaire, saisir un texte et le corriger, envoyer un message en utilisant une plateforme électronique pour communiquer avec les chercheurs lors des clavardages). Ces formations sont dispensées par des chercheurs de la CUDC qui effectuent des missions de travail sur le terrain au Sénégal où a lieu la recherche-action. Les chercheurs de la CUDC travaillent conjointement avec des chercheurs sénégalais de l'INÉADE. Dans ce projet, les TIC sont exploitées dans une perspective socioconstructiviste et interactive à travers une mise en contexte des savoirs scolaires.

Dans la phase de coconstruction de situations (phase 2), les chercheurs et les enseignants expérimentateurs collaborent dans la construction de situations d'enseignement-apprentissage et d'évaluation (SEA/É) axées sur l'intégration des TIC aux activités de la classe dans le but précis de développer des dispositifs didactiques qui transforment les pratiques pédagogiques des enseignants. Ces situations d'apprentissage sont conçues à partir des thèmes choisis consensuellement entre élèves et enseignants-expérimentateurs. Aidés par le chercheur de la CUDC, les enseignants planifient des tâches et des activités axées sur un apprentissage efficace de la lecture, de l'écriture et de l'expression orale par des élèves. Ces situations d'apprentissage sont validées par le chercheur de la CUDC (un didacticien du français) et son homologue du Sénégal. L'ingénierie didactique mise en place vise à développer des compétences en littératie, concept qui est compris, comme l'écrit Pierre (2003) par : « [l]e fait de maitriser l'écrit pour pouvoir penser, communiquer, acquérir de nouvelles connaissances, résoudre des problèmes, réfléchir sur notre existence, partager notre culture ou se distraire est ce qui définit le type et le niveau de littératie atteint par des individus que l'on dira lettrés - litterati - au sens où l'entendait Cicéron qui posait la littératie - scientia litteratura - à la fois comme le fondement de la sagesse et de l'éloquence (Pattison, 1982)».

Durant la phase 3 consacrée à l'accompagnement didactique, les situations coconstruites sont mises en œuvre en classe. Ensuite, des données sont recueillies lors des observations de séquences d'enseignement-apprentissage du français en $5^{e}$ année et durant des entrevues réalisées auprès de deux enseignants expérimentateurs en utilisant un canevas d'entretien à cinq questions (annexe 2). Le contenu des journaux de bord complétés durant les observations est validé lors des focus groups, généralement consécutifs aux séquences d'enseignement-apprentissage et lors des entrevues menées sous forme d'entretiens d'explicitation (Vermesh, 1994). Les focus groups sont animés par le chercheur CUDC à l'intention de l'enseignant expérimentateur, du directeur de l'école SAAM et à l'intention d'un groupe de chercheurs de l'INÉADE (un spécialiste du français, un spécialiste en gestion de classe, un technologue). Les entrevues avec les enseignants et les échanges lors des focus groups sont enregistrés sur vidéo par un technologue de la CUDC. L'analyse du contenu (Bardin, 1986) est utilisée pour traiter toutes ces données dont nous présentons ci-après les résultats. 


\section{Résultats et commentaires}

Dans une perspective socioconstructiviste, les savoirs enseignés en classe de français, langue de scolarisation à l'école SAAM, tiennent compte du contexte socioculturel, économique et politique, de la situation d'apprentissage (avec ses contraintes et ses ressources) ainsi que des préoccupations et des représentations de l'élève, de ses dispositions, des ressources disponibles et de ses connaissances antérieures sur l'objet d'apprentissage qu'est le français (langue seconde ou troisième chez certains élèves). Ainsi, les activités d'apprentissage proposées dans les projets intégrateurs (PI) ont permis à l'élève de donner du sens à ses apprentissages scolaires. Voici un témoignage d'un enseignant expérimentateur à ce sujet : «Avant que l'on aille chez le menuisier, les élèves ne savaient pas ce que c'est! Donc, la sortie chez le menuisier nous a permis d'engranger, d'enrichir le vocabulaire. On a eu un vocabulaire qu'on a exploité en classe. Par exemple, scier, raboter, la sciure, les copeaux... Eh! C'est des mots que les élèves ignoraient ou ne connaissaient pas. Lorsqu'on est revenus en classe, on a mis ça dans une leçon de vocabulaire, on a exploité ».

L'utilisation des outils numériques à différents moments des séquences didactiques des PI permet une médiation des différents savoirs visés par l'enseignement du français, langue de scolarisation. Ces outils numériques sont de puissants outils psychologiques au service de l'apprentissage de la langue. Ce sont des artéfacts qui facilitent l'adhésion au projet de la classe, de la communauté apprenante. Ils créent un environnement informatique qui est considéré comme un outil à potentiel cognitif (OPC) (Karsenti et Collin, 2013). L'expérience menée à l'école pilote SAAM montre que les outils numériques sont des ressources importantes pour le développement des compétences langagières chez les élèves. Le développement de leurs habiletés et de leurs capacités langagières se fait à travers des situations d'apprentissage planifiées en contexte (le marché, la fête de la Tabaski, le jardin scolaire, etc.). Ainsi que l'affirme un enseignant au CM1, les enquêtes menées sur le terrain, à l'extérieur des murs de l'école, permettent aux élèves de coopérer et de réaliser ensemble des apprentissages signifiants en français en utilisant la technologie. Voici son témoignage : «Lorsque nous avons été au marché, lorsque également nous avons été au quai de pêche, les enfants sont scindés en groupes. Ils ont exploré donc ces milieux, avec leur appareil (...), prise de photos. Puis, ils sont scindés en trois groupes. Sur la base justement de eeeh! D’un questionnaire que nous avons préparé en classe. En tous cas, ils ont posé les questions auxquelles ils voulaient avoir des réponses. On trouve que ce qui les intéressait au marché, c'est les ordures, l'insalubrité et cela a débouché sur la production de textes de type informatif. En tous cas, le compte rendu de la visite au marché a généré des comptes rendus différents parce que s'appuyant sur des supports n'est-ce pas différents, et des canaux différents, portraits, description et textes informatifs ». 
Comme l'écrivent Brown, Collins et Duguid (1989), activité et contexte sont parties intégrantes de la cognition et de l'apprentissage au sein d'une communauté de pratique. Pour ces auteurs, le concept de communauté de pratique réfère au fait que le sujet humain n'apprend jamais seul et qu'il appartient toujours à une communauté avec laquelle il se coproduit. Ainsi que l'affirment plusieurs études, les pratiques de la communauté constituent le savoir de la communauté et c'est en prenant part à la vie de celle-ci que le sujet accède à certaines formes de compétences qui sont toujours des propriétés distinctives du groupe (Lave et Wenger, 1989; Wenger, 1998). Dans cette perspective théorique, le savoir (lire, écouter, parler et écrire) ne peut pas être indépendant des situations qui sont imbriquées dans le contexte de l'école sénégalaise et dans la communauté d'apprentissage à l'école SAAM. Par ailleurs, l'intégration de la technologie dans les apprentissages de base permet d'introduire des innovations pédagogiques où l'outil psychologique est au cœur d'une expérience qui vise à stimuler des interactions entre élèves d'une part, et entre élèves et l'enseignant d'autre part. Voici un exemple qui illustre la nature des interactions portant sur la pertinence de l'ordinateur en salle de classe. Les propos rapportés proviennent d'une séquence d'enseignement filmée en classe de CE2 :

Enseignante : Savoir utiliser les ordina...

Élèves (en cœur) : ....teurs.

Enseignante: Très bien! Et quoi encore? (Elle pointe vers un élève). Toi.

Élève (désigné par l'enseignante) : Savoir photographier.

Enseignante: Quoi encore? (Elle pointe vers un autre élève).

Élève (désigné par l’enseignante) : Savoir insérer des images.

Enseignante: Quoi encore? (Elle pointe vers un autre élève).

Élève (désignée par l'enseignante) : Savoir copier-coller.

Enseignante : Est-ce qu'avant vous le saviez?

Élèves (en cœur) : Non.

Enseignante: Vous ne le saviez pas! Très bien. Et quoi encore? (Elle pointe vers un élève).

Élève (désigné par l'enseignante) : Savoir élever un animal.

Enseignante: Très bien. Quoi encore? (Elle pointe vers un élève).

Élève (désigné par l'enseignante) : Comment on le soigne. 
Le contenu de ces échanges entre les élèves et l'enseignante témoigne de l'importance accordée à la technologie par ces nouveaux utilisateurs. De plus, dans une approche pédagogique axée sur des situations de vie, l'outil cognitif inscrit le savoir dans un rapport instrumental par rapport à l'action de l'élève. Ainsi, les diverses ressources multimodales qu'offrent certaines applications de l'ordinateur (traitement de texte, lecture à l'écran, préparation d'un PowerPoint, clavardage, envoi de courriels, etc.) transforment et présentent les savoirs essentiels visés par l'enseignement du français langue de scolarisation sous un autre jour. Qu'il s'agisse des textes lus directement à l'écran, des images ou des vidéoclips téléchargés de l'Internet ou des textes variés qui sont produits en équipe ou individuellement grâce au traitement de texte, les savoirs prescrits dans le programme d'études sont présentés à l'élève sous des formes variées. Cette variété dans la façon d'accéder au savoir (variété sous forme d'image, sous forme sonore ou audio, sous forme de texte continu, sous forme de schéma ou d'illustration, etc.) facilite l'apprentissage de la langue à l'école pilote de Dakar. L'innovation technologique contribue à la participation active des élèves et à leur construction sociale du savoir à travers des activités qui reculent les murs de la classe traditionnelle (Defise et Simbagoye, 2008; Simbagoye, Guèye, Dog et Sougou, 2010).

Pour les enseignants-expérimentateurs, l'amélioration de l'expression orale et un accroissement du vocabulaire acquis sont constatés lors des activités individuelles ou dans des tâches collaboratives. L'analyse de contenu des observations et des productions écrites des élèves (textes écrits et portfolios d'apprentissage) a montré l'importance des acquisitions relatives aux notions linguistiques et textuelles chez les élèves des classes expérimentales CM1 et CM2. Comme l'écrit Crinon (2006) au sujet de l'écriture, un des enjeux majeurs de la didactique est de permettre aux élèves de se construire des représentations pertinentes de l'écriture. D'ailleurs, des études menées en France et ailleurs dans le monde montrent que les technologies et les médias constituent des aides importantes à l'écriture chez des jeunes scripteurs (Crinon, 2006). Selon l'équipe de recherche Coditexte, la mise à la disposition des élèves d'une base de textes d'auteurs leur permet d'améliorer et d'enrichir leurs propres écrits à condition qu'ils les consultent sur un écran et qu'ils y accèdent à l'aide des mots-clés.

Que ce soit à l'oral ou en lecture, lors de la révision en rédaction ou lors de l'évaluation des textes des pairs, les interactions entre les élèves prouvent qu'ils recourent aux mécanismes d'analyse perceptive - décodage - et aux mécanismes d'analyse conceptuelle - compréhension - (Pierre, 2003) qui avaient été préalablement enseignés. Pour l'un des enseignants-expérimentateurs, les élèves de l'école ne travaillent pas sur des situations théoriques à la manière des grammairiens ou des linguistes. Ils traitent des situations signifiantes et qui concernent leur vécu. Ils construisent la signification des textes qu'ils lisent en faisant des inférences à partir des illustrations, à partir des mots qu'ils ne connaissaient pas avant en utilisant des ressources de l'Internet (voir Annexe1). De fait, la compréhension des écrits est une recherche du sens qui recourt habituellement au décodage, au vocabulaire global (mot facilement identifiable par le lecteur) et au contexte linguistique (Giasson, 2003). Lors des échanges en travail de groupe, les élèves recourent aux processus de la pensée critique et réflexive, ingrédients fondamentaux du processus de développement des compétences en littératie. À l'écrit, 
ils ont manifesté des habiletés de planification, de mise en texte, de révision et de publication. Or, les activités rédactionnelles sont considérées par les recherches en didactique comme des processus de haut niveau (par exemple, l'évaluation critique d'un texte, le fait de faire des inférences, le résumé d'un récit) (Crinon, 2006). Et quelle place occupent les outils technologiques? Par leurs actions de recherche d'informations et de correction des textes produits, les élèves recourent aux processus de bas niveau lorsqu'ils utilisent les fonctionnalités de l'ordinateur (par exemple, taper un mot, repérer une image, trouver un synonyme). Dans les textes rédigés en équipe, les élèves appliquent des règles qui étaient auparavant inopérantes, comme en témoignent les faibles taux de réussite aux épreuves d'évaluation cantonales et nationales des années antérieures au projet TIC. Lors des échanges par courriel, des mots nouveaux appris sont utilisés par des élèves qui n'ont pas l'habitude de s'exprimer en français, leur langue seconde ou leur troisième langue apprise à l'école. En évaluant les productions des pairs, ils décodent et interprètent un texte afin d'identifier des erreurs d'orthographe, d'accord, ils relèvent des mots mal utilisés et toute autre source de malentendu communicatif qu'ils justifient en rédigeant un commentaire explicatif d'une cote attribuée au travail des pairs : bien, assez bien ou à améliorer.

L'approche didactique mise de l'avant par le projet remet l'apprenant au centre de l'action en situation d'apprentissage, autant en classe qu'à l'extérieur des murs de la classe ou de l'école. Il promeut une dynamique des activités langagières dans des situations d'enseignement et d'apprentissage qui font appel aux TIC, mais dans des contextes ou des environnements connus de l'élève. 
Figure 1. Illustration de la démarche intégrant la technologie dans des activités en classe de français

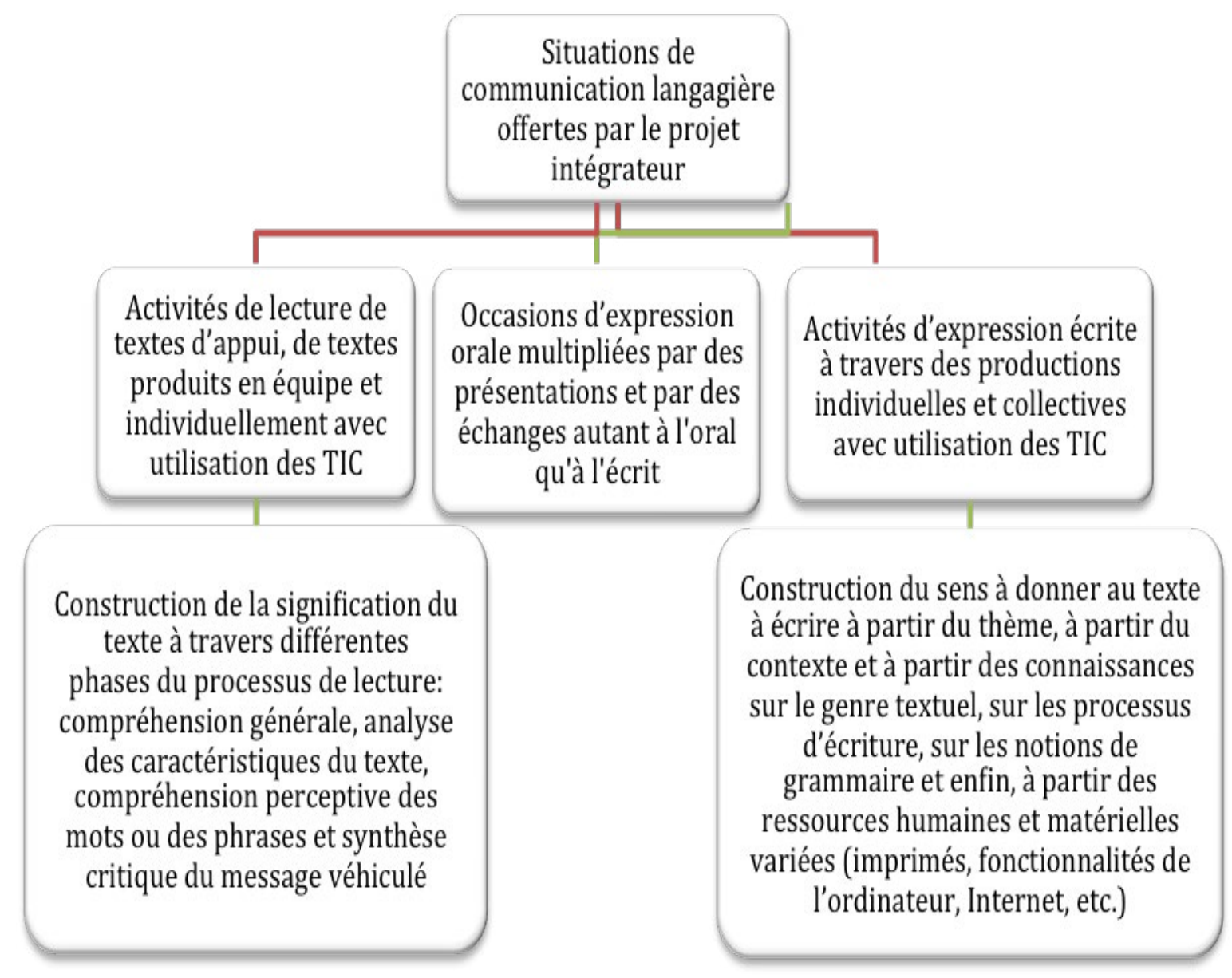

L'outil technologique est à la fois un objet d'enseignement, mais aussi une ressource qui permet à l'élève d'être au centre de la situation didactique. Il guide le traitement cognitif de la tâche et facilite les échanges et la communication de données par des symboles variés (mots, textes, images, illustrations, vidéo, schémas et autres productions médiatiques variées) en situation (Legros, Crinon et Martin, 2006; Picardo, 2005). Avec les TIC, les élèves sont amenés à traiter des situations d'apprentissage de façon motivante. Les observations des séquences et les entrevues menées avec les enseignants montrent que l'élève est motivé, qu'il est actif et, surtout, qu'il veut « jouer » avec la technologie. Un des enseignants au CM1 ( $5^{e}$ année) rappelle « qu'un enfant qui joue est plus sérieux qu'un chercheur dans son laboratoire (...) le facteur déterminant, c'est la motivation. Parce que l'ordinateur, coonsi... un élément nouveau pour l'enfant ». Quant à Mme N., une enseignante expérimentatrice au CE2, elle affirme ceci : «Avant, l'enfant ne produisait qu'en CM1. En CE2, il faisait que de courtes phrases. Maintenant, il est obligé de parler, il est obligé d'écrire. Parce qu'à chaque fois, il faut qu'il écrive ce qu'il a fait, ce qu'il a trouvé. Donc ça pousse l'enfant à écrire. Tout ce qu'il fait l'oblige à écrire. Parce que tout ce qu'il fait il doit l'écrire. Il ne doit pas rester là, il doit faire, il doit chercher, il doit (...). Il est obligé de travailler. C'est pour cela je dis : voilà, voilà des élèves faibles. Ce sont des enfants faibles, 
mais ils manipulent l'ordinateur. Ils savent ouvrir, ils savent chercher, ils savent où il faut aller, trouver ce qu'ils cherchent. Ce sont des élèves faibles. Seulement, la difficulté, c'est de lire ce qu'ils trouvent. Là il faut que tu les orientes. Avec les TIC, l'enfant est obligé de travailler et ça les intéresse ».

En plus de servir de ressource de motivation et de stimulation de l'action d'apprentissage, la technologie est une ressource importante au service de l'action enseignante, comme le souligne un autre enseignant expérimentateur : «L'utilisation des TIC change tout, hein! Pratiquement. Dans la façon de mener nos leçons, dans l'exécution, dans... dans la façon de préparer, la démarche même peut changer. Parce que euh! (...) Bon, je eee, je dis aux élèves, demain on a une leçon SVT, sciences de la vie et de la terre, par exemple, sur la poule. Les élèves anticipent. Eh! Ils anticipent. Ils vont recueillir toutes les informations que leur machine leur donne. Arrivés en classe, vous allez directement à l'ordinateur, ce sont les élèves qui vont vous enlever la poule. Hein! Hein! Ils vous enlèvent directement la poule et vous regardez, dans l'ordinateur on peut avoir le schéma de la poule. Donc, ils ont le schéma de la poule. Ils peuvent l'annoter. Vraiment, l'enseignement devient beaucoup plus facile ». Grâce à la technologie, les élèves de l'école pilote, qui n'avaient aucune tradition du livre, développent une idée claire de ce qu'est l'écrit par le biais de genres textuels à l'étude : les types de textes explicatifs et descriptifs à propos des thèmes étudiés tels que rapportés par les enseignants interviewés (par exemple, «jardin scolaire», « au marché »).

\section{Émergence d'un modèle didactique holistique}

L'approche par les situations adoptée par les chercheurs de la CUDC oriente la formation des enseignants expérimentateurs vers le développement de compétences professionnelles et non pas seulement vers la transmission des contenus à mémoriser et vers la maitrise des gestes à répéter (Munger et Jonnaert, 2007; Cyr, Defise, Gueye, Jonnaert, Munger, Sene, Simbagoye et Sougou, 2008; Simbagoye, Jonnaert, Defise et Cossi, 2012). Dans le cadre des PI avec intégration des TIC, la technologie a servi de médiateur de l'action réflexive de l'élève. L'hypothèse socioconstructiviste est à la base d'un modèle qui po stule que le sujet construit ses connaissances en situation et en collaboration avec les pairs, mais également avec l'enseignant. Les connaissances construites par l'élève, mis dans des situations didactiques intéressantes motivantes, et ce, grâce à l'intégration des TIC, constituent une ressource fondamentale. Cette ressource lui permet d'être compétent dans ces mêmes situations. Ces situations sont donc déterminantes, car ce sont elles qui permettent à l'élève d'utiliser ses connaissances viables pour se montrer compétent en situation. L'idée de situation sous-tend l'existence d'un contexte global (social, culturel, politique ou économique de Dakar). De là viennent les trois dimensions (constructiviste, interactive et socioconstructive) consubstantielles d'une approche qui permet de dégager un modèle didactique holistique permettant la construction de l'univers langagier chez l'élève à travers l'intégration des TIC dans les projets intégrateurs. 
D’abord, la dimension constructiviste (C) évoque l'activité du sujet qui porte sur les idées, les connaissances et les conceptions que le sujet possède déjà à propos de l'objet à apprendre. L'élève en situation d'apprentissage de la langue d'enseignement construit ses connaissances langagières par une activité réflexive sur ce qu'il sait déjà, adaptant ses propres connaissances aux exigences de la situation à laquelle il est confronté et aux caractéristiques qu'il décode lui-même sur les savoirs prescrits en français. La dimension constructiviste évoque donc l'activité réflexive de l'élève sur ses propres connaissances et sur les opérations qu'il effectue sur les savoirs qui sont codifiés dans les manuels scolaires et les autres ressources comme les dictionnaires électroniques. Dans le cadre des PI avec intégration des TIC, la technologie a servi de médiateur de l'action réflexive de l'élève. Ensuite, la dimension interactive (I) fait référence aux situations auxquelles l'élève est placé et où ses connaissances actuelles se heurtent à un nouvel objet à apprendre. La dimension « interactive » alimente les activités réflexives et dialectiques de la dimension constructiviste. De manière fonctionnelle, les dimensions constructive et interactive fonctionnent solidairement, l'une n'étant pas possible sans l'autre. Le didacticien de la langue trouve donc dans cette dimension à la fois constructive et interactive la référence à un objet à apprendre qui appartient aux quatre catégories d'action en classe de langue : écouter, parler, lire et écrire. Dans une approche textuelle de l'enseignement de la langue, ces quatre catégories d'action unifient les trois volets de l'enseignement de la langue, à savoir la lecture, l'écriture et l'oral. Enfin, la dimension Socio (S) est relative aux interactions sociales qui constituent une composante essentielle du processus de construction des connaissances en classe de français. En effet, les interactions sociales suscitent des conflits sociocognitifs intra-individuels. Elles sont faites des échanges avec les autres élèves (de sa classe ou non) et des échanges avec les adultes de l'école ou de la communauté au sein d'une culture donnée.

Avec le modèle $\mathrm{SCl}$, les enseignants sont amenés à réfléchir sur leur pratique de classe dans le cadre d'un dispositif d'accompagnement à travers des forums de discussion à distance et lors des focus groups qui suivent des observations de séquences d'enseignement-apprentissage en salle de classe. L'approche didactique mise de l'avant par le projet de recherche-action remet l'apprenant au centre de l'action en situation d'apprentissage, autant en classe qu'à l'extérieur des murs de la classe ou de l'école. Il promeut une dynamique des activités langagières dans des situations d'enseignement et d'apprentissage qui font appel aux TIC, mais dans des contextes ou des environnements connus de l'élève (Munger et Jonnaert, 2007). Comme l'esquisse la figure 2 ci-après, le dispositif pédagogique mis en place par coconstruction de situations débouche sur une interdépendance des activités langagières à travers l'articulation des activités de lecture, d'expression orale et de production écrite avec médiation de la technologie (Defise et Simbagoye, 2008; Simbagoye et al., 2010; Simbagoye, 2012). 
Figure 2. Modèle $\mathrm{SCl}$ avec intégration de la technologie en classe de français

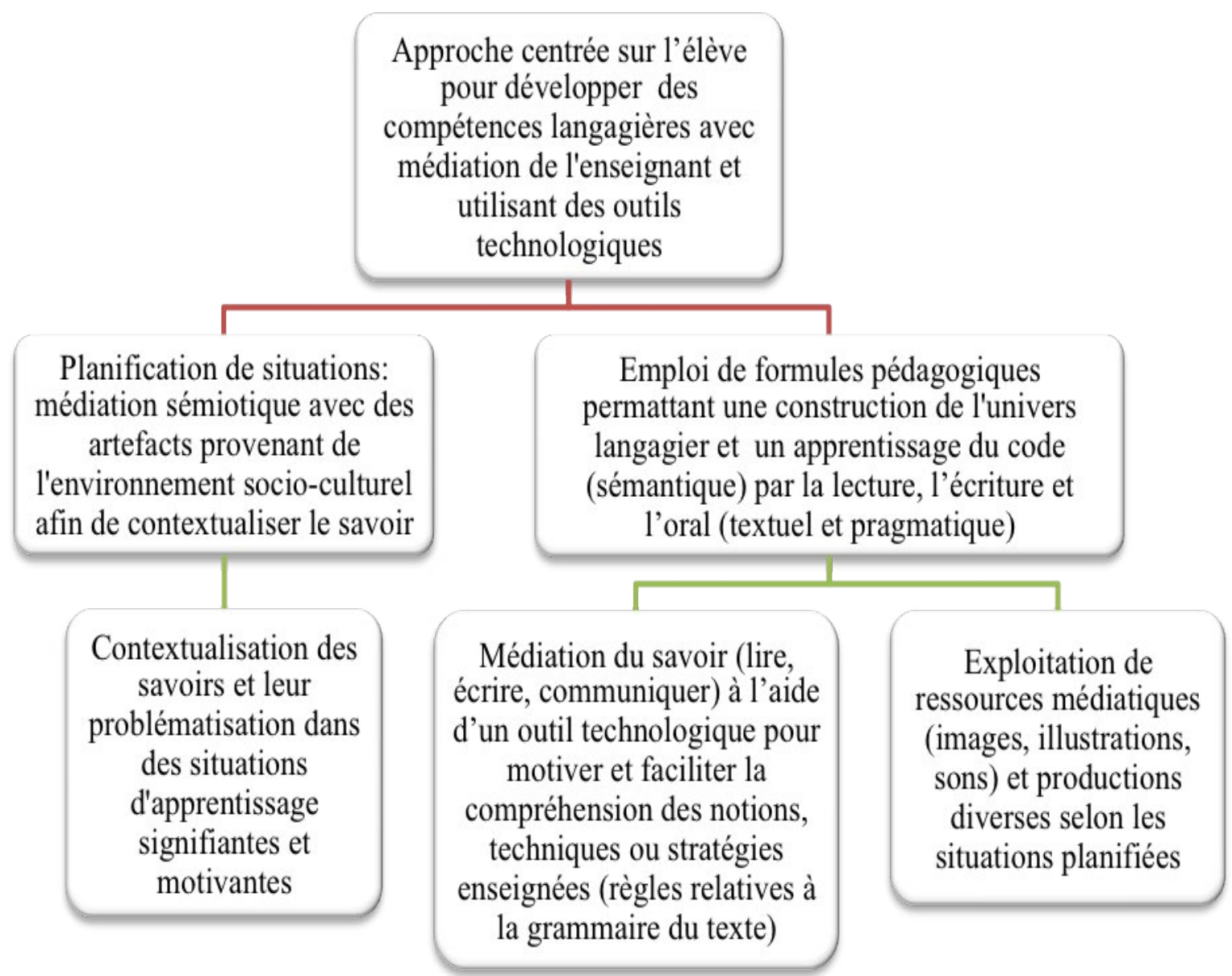

\section{Conclusion}

L'objectif de ce texte est de montrer que l'intégration des TIC dans les apprentissages en français contribue à l'amélioration de l'enseignement-apprentissage. Elle contribue également à modifier le rapport de l'élève à l'écrit, car ses représentations et son attitude envers l'écrit changent positivement. Cela s'observe à travers plusieurs éléments positifs : utilisation efficiente des paramètres de la situation de communication, prise en compte des contraintes de l'orthographe (lexical et grammatical), respect du sens généralement attribué aux mots utilisés, présence d'indices textuels enseignés (organisateurs textuels, marqueurs de relations, emploi de pronoms pour assurer la progression de l'information dans le texte, etc.). À la lumière des résultats de cette recherche-action, le constat est que les situations planifiées en équipe (enseignant-chercheur) débouchent sur une interdépendance des activités langagières à travers l'articulation des activités de lecture, d'expression orale et de production écrite. De plus, des apprentissages informels sont réalisés : l'appropriation de la recherche par requête dans le Web (Google, etc.), l'envoi de messages par courriel ou par téléphone, la capacité de travailler en équipe et l'engagement accru lors des activités de compréhension en lecture, d'écri- 
ture et de discussion en français. Bref, les situations planifiées dans le cadre du projet d'intégration des TIC ont permis une construction de l'univers langagier chez l'élève. II serait intéressant que des recherches soient menées pour documenter la portée de tels apprentissages en analysant, par exemple, le niveau de rendement des élèves lors des évaluations à grande échelle. Également, on pourrait comparer les performances des élèves du cycle moyen provenant d'une même région, répartis en deux catégories : un groupe d'élèves d'une école utilisant les TIC et un autre groupe d'élèves inscrits dans une école n'ayant pas encore adopté l'usage des TIC en salle de classe. 


\section{Références}

Bardin, L. (1986). L'analyse de contenu (4éd.). Paris, France: Presses universitaires de France.

Brown, J. S., Collins, A. et Duguid, P. (1989). Situated cognition and the culture of learning. Educational Researcher, 18(1), 32-42.

Crinon, J. (2006). Lire et écrire la fiction: quelques malentendus. Repères, 33, 61-79.

Cyr, S., Defise, R., Gueye, I., Jonnaert, P., Munger, P., Sene, P., Simbagoye, A. et Sougou, M. (2008). Profil de sortie de la formation des enseignantes et des enseignants intégrant les TIC dans leur pratique quotidienne. Dakar, Sénégal : INÉADE-ORÉ.

Defise, R. et Simbagoye, A. (2008). Gestion de classe et acquisitions en français dans un processus d'intégration des TIC dans les apprentissages de base d'élèves d'une école élémentaire au Sénégal. Dakar, Sénégal : Éditions des Écoles Nouvelles africaines.

Giasson, J. (2003). La lecture. De la théorie à la pratique (3e éd.). Boucherville, Québec : Gaëtan Morin éditeur.

Jonnaert, P. (2010). Intégration des TIC dans les apprentissages de base à l'école élémentaire. Montréal : Chaire UNESCO de Développement Curriculaire de l'Université du Québec à Montréal (CUDC)/Université du Québec à Montréal (UQAM).

Jonnaert, P. (2004). Adaptation et non transfert. Dans P. Jonnaert et D. Masciotra (dir.), Constructivisme: Choix contemporains. Hommage à Ernst Von Glasersfeld (p. 197-204)., Sainte-Foy, Québec: Presses de l'Université du Québec.

Karsenti, T. et Collin, S. (2013). TIC et éducation : avantages, défis et perspectives futures. Éducation et francophonie, XL/(1), 1-6. Repéré à http://www.acelf.ca/c/revue/pdf/EF-41-1-001_liminaire. pdf

Lave, J. (1991). Acquisition des savoirs et pratiques de groupe. Sociologie et sociétés, 23(1), 145-162.

Lave, J. et Wenger, E. (1991). Situated learning : legitimate peripheral participation. Cambridge: University of Cambridge.

Legros, D., Crinon, J. et Marin, B. (2006). Réécrire et apprendre à réécrire : le rôle d'une base de données textuelles. Langages, 164, 98-112.

Maturana, R. H. (1998). Reality: The search for objectivity or the quest for a compelling argument. The Irish Journal of Psychology, 9(1), 25-82.

Munger, P. et Jonnaert, P. (2007). Conditions et effets de l'implantation des TIC dans un processus d'intégration des TIC dans les apprentissages de base d'élèves d'une école élémentaire au Sénégal. Dakar, Sénégal : Éditions des écoles nouvelles africaines (EENAS).

Pierre, R. (2003). Entre alphabétisation et littératie : les enjeux didactiques. Revue française de linguistique appliquée, 1(8), 121-137. 
Roth, W.-M. et Masciotra, D. (2004). Apprendre, c'est faire émerger. Dans P. Jonnaert et D. Masciotra (dir.), Constructivisme: Choix contemporains. Hommage à Ernst Von Glasersfeld (p. 225-253). Sainte-Foy, Québec: Presses de l'Université du Québec.

Simbagoye, A., Jonnaert, P., Cossi, C. et Defise, R. (2012). Manuel à l'usage des formateurs (trices) d'ENI : un cadre d'action pour la formation des enseignant(e) s au primaire. Washington : Educational Center Development-USAID-Bénin.

Simbagoye, A., Chartrand, P., Daviau, C. et Cyr, S. (2012). Écoles en mouvement et réformes : enjeux, défis et perspectives - États des lieux et questions curriculaires. Bruxelles, Belgique : De Boeck.

Simbagoye, A., Gueye, I., Dog, S. et Sougou, M. (2010). L'intégration des TIC, une voie prometteuse dans les apprentissages du français à l'école élémentaire. Dakar : INÉADE-ORÉ.

Von Glasersfeld, E. (2004). Introduction à un constructivisme radical. Dans P. Jonnaert et D. Masciotra (dir.), Constructivisme : Choix contemporains. Hommage à Ernst Von Glasersfeld (p. 12-36). Sainte-Foy: Presses de l'Université du Québec.

Von Glasersfeld, E. (1999). Pourquoi le socioconstructivisme doit-il être radical? Revue des sciences de l'éducation, 20(2), 21-29.

Vygotsky, L. S. (1985). Pensée et langage. Paris, France : Éditions sociales.

Wenger, E. (1998). Communities of practice : learning, meaning and identity. Cambridge : Cambridge University Press.

Vermersh, P. (1994). L'entretien d'explicitation en formation initiale et continue. Paris, France : ESF. 


\section{Annexe}

\section{Annexe 1. Exemple de séquence observée au CM1 et ayant fait l'objet de focus groupe entre enseignants expérimentateurs et deux chercheurs de la CUDC}

\begin{tabular}{|c|c|}
\hline Unité & - Texte explicatif (Thème: Jardin scolaire). \\
\hline But & $\begin{array}{l}\text { - Analyser le texte de manière critique et ressortir des procédés linguistiques et textuels permettant de véhiculer le } \\
\text { sens d'un message. }\end{array}$ \\
\hline \multicolumn{2}{|l|}{ Moments didactiques } \\
\hline Contextualisation & $\begin{array}{l}\text { - Rappel de l'importance des textes de type descriptif et explicatif (décrire ou expliquer une réalité, afin de la faire } \\
\text { comprendre à une personne). } \\
\text { - Questionnement des élèves sur l'importance de la phrase impérative et rappel que l'utilisation de types de phrase } \\
\text { (déclarative, interrogative, impérative et exclamative) et de formes variées (active, passive, neutre ou emphatique) } \\
\text { affecte le message. } \\
\text { - Explication du but de l'activité et des tâches (particularités de la phrase affirmative et des marques d'organisation } \\
\text { textuelle). }\end{array}$ \\
\hline Décontextualisation & $\begin{array}{l}\text { - Lecture de texte - lecture silencieuse, lecture expressive de l'enseignant et de quatre élèves; } \\
\text { - Réaction au texte (compréhension) et partage d'expérience (quelques élèves racontent leur expérience). Consulta- } \\
\text { tion du dictionnaire de français pour vérifier le sens des mots nouveaux. } \\
\text { - Observation du phénomène (en équipe de } 5 \text { à } 6 \text { élèves) : utilité de la phrase déclarative sous ses différentes formes } \\
\text { et inventaire des marques d'organisation graphique et textuelle (marqueurs de relation). } \\
\text { - Manipulation des énoncés en comparant les différentes phrases d'un paragraphe du texte choisi et en relevant les } \\
\text { marques de relation dans deux textes. } \\
\text { - Formulation des hypothèses sur les notions à l'étude par les élèves et mise en commun pour leur validation. } \\
\text { - Enseignement explicite des notions à l'étude : formes de phrases et marques d'organisation graphique et textuelle } \\
\text { (marqueurs de relation). }\end{array}$ \\
\hline Recontextualisation & $\begin{array}{l}\text { - Relecture des textes descriptifs écrits en équipe et autocorrection en insistant sur la vérification de l'utilisation des } \\
\text { formes de phrases et des marques d'organisation du texte. } \\
\text { - Échanges de textes et leur évaluation par les pairs. } \\
\text { - Formulation de commentaires et mise en commun en grand groupe. } \\
\text { - Envoi des commentaires de chaque groupe par l'élève responsable à l'enseignant expérimentateur. }\end{array}$ \\
\hline
\end{tabular}




\section{Annexe 2. Canevas d'entrevue avec les enseignants expérimentateurs}

1. Comment la situation d'enseignement-apprentissage planifiée permet-elle de générer des apprentissages riches pour l'acquisition de la langue d'enseignement dans votre classe?

2. Expliquez comment les tâches réalisées par les élèves tiennent compte de leurs besoins et de leurs connaissances antérieures en français.

3. Quelles sont les activités (travail individuel ou travail fait en équipe) qui montrent que vous avez créé un environnement de travail où l'élève est actif? (nommez les actions posées par les élèves)

4. Quelles sont les stratégies ou formules pédagogiques ayant été utilisées pour amener l'élève à intégrer la technologie dans les trois volets de la langue?

5. Quelle importance avez-vous accordée aux réalisations (individuelles ou collectives) des élèves? 